JOURNAL OF SYNCHROTRON RADIATION

ISSN 1600-5775

\section{Hard X-ray-induced damage on carbon-binder matrix for in situ synchrotron transmission $X$-ray microscopy tomography of Li-ion batteries}

\author{
Cheolwoong Lim, ${ }^{a}$ Huixiao Kang, ${ }^{a}$ Vincent De Andrade, ${ }^{b}$ Francesco De Carlo ${ }^{b}$ and \\ Likun Zhu ${ }^{\mathrm{a} *}$
}

Received 12 December 2016

Accepted 23 February 2017

Edited by J. F. van der Veen

Keywords: radiation damage; Li ion battery; in situ; carbon-binder matrix; intermittent dose.

Supporting information: this article has supporting information at journals.iucr.org/s
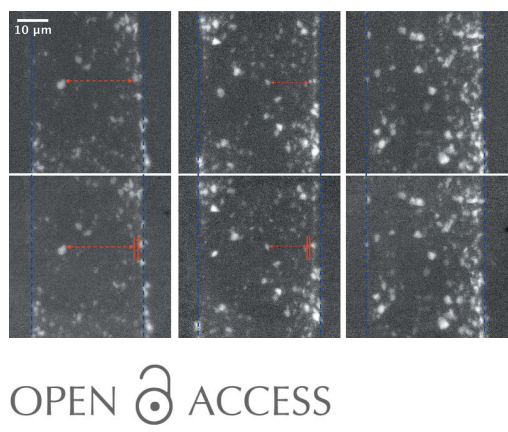

aDepartment of Mechanical Engineering, Indiana University-Purdue University Indianapolis, Indianapolis, IN 46202, USA, and ${ }^{\mathbf{b}}$ Advanced Photon Source, Argonne National Laboratory, Argonne, IL 60439, USA.

*Correspondence e-mail: likzhu@iupui.edu

The electrode of Li-ion batteries is required to be chemically and mechanically stable in the electrolyte environment for in situ monitoring by transmission $\mathrm{X}$-ray microscopy (TXM). Evidence has shown that continuous irradiation has an impact on the microstructure and the electrochemical performance of the electrode. To identify the root cause of the radiation damage, a wire-shaped electrode is soaked in an electrolyte in a quartz capillary and monitored using TXM under hard X-ray illumination. The results show that expansion of the carbon-binder matrix by the accumulated X-ray dose is the key factor of radiation damage. For in situ TXM tomography, intermittent X-ray exposure during image capturing can be used to avoid the morphology change caused by radiation damage on the carbon-binder matrix.

\section{Introduction}

Transmission X-ray microscopy (TXM) is an image acquisition technique that captures transmitted monochromatic X-rays through a sample. Recently, the microstructure of porous electrodes of Li-ion batteries (LIBs) has been reconstructed using TXM tomography by collecting a series of TXM images during a $180^{\circ}$ rotation of the electrode (Lim et al., 2016). The non-destructive advantage of TXM is that it allows the study of the in situ morphology change of high-capacity LIB anode materials, such as Ge (Weker et al., 2014) and Sn (Wang et al., 2014). During in situ TXM tomography, the electrode is exposed under a high radiation dose which can cause radiation damage on the electrode. Thus, it is necessary to investigate the radiation-induced damage for in situ TXM tomography. Previous studies have shown radiation damage on the LIB electrode (Weker et al., 2014) during in situ TXM. However, the root cause of radiation damage is still elusive because all the components of a working LIB cell could be impacted by $\mathrm{X}$-rays and any damage on any component could affect the performance of the cell significantly. Nelson et al. (2013) have shown that irradiated sulfur particles were dissolved in electrolyte after $20 \mathrm{~s}$ of $6 \mathrm{keV}$ X-ray exposure. Irradiation of an electrode could also affect the carbon-binder matrix because polymers are sensitive to X-ray irradiation (Coffey et al., 2002; Vaselabadi et al., 2016). The objectives of this paper are to investigate the hard X-ray induced damage on the carbonbinder matrix in in situ LIB cells and to find ways to avoid the damage. 


\section{Experimental}

A wire-shaped electrode was sealed in a $500 \mu \mathrm{m}$ quartz capillary tube (Hampton Research, Aliso Viejo, CA, USA) which was filled with $1 M \mathrm{LiPF}_{6}$ in EC/DEC electrolyte $(1: 1$ volume-ratio mixture of ethylene carbonate and dimethyl carbonate, BASF, USA). The wire-shaped electrode was fabricated by coating electrode slurry on a $30 \mu \mathrm{m}$-diameter carbon wire (WPI, Sarasota, FL, USA). The slurry was composed of $50 \mathrm{wt} \%$ active material $\left(\mathrm{Ge}_{0.9} \mathrm{Se}_{0.1}\right), 30 \mathrm{wt} \%$ conductive carbon (C65, TIMCAL) and $20 \mathrm{wt} \%$ carboxymethyl cellulose binder (Sigma-Aldrich, pre-dissolved in deionized water). The micrometer-sized $\mathrm{Ge}_{0.9} \mathrm{Se}_{0.1}$ particles were provided by C. B. Mullins's group (Klavetter et al., 2015). The quartz capillary housing and carbon-wire current collector were selected because of their low X-ray attenuation. TXM tomography was implemented under an X-ray energy of $11.2 \mathrm{keV}$ at the beamline 32-ID-C of the Advanced Photon Source (APS). The photon rate in a field of view of around $74.6 \mu \mathrm{m}$ is approximately $1.17 \times 10^{11}$ photons s$^{-1}$ at $11.2 \mathrm{keV}$ at this beamline. The illumination at the edges of the field is weaker, which results in lower radiation dose at the edges of the field of view. The wire electrode is located at the center of the field view and is about $39.1 \mu \mathrm{m} \times 60.1 \mu \mathrm{m}$ in the projection image. The photon rate in this area is approximately $7.12 \times$ $10^{10}$ photons s $^{-1}$, which is calculated from the intensity profile of a flat-view image. It should be noted that the radiation intensity is reduced by approximately $2.8 \%$ and $11.7 \%$ before reaching the wire electrode by the $10 \mu \mathrm{m}$-thick wall of quartz capillary and the $200 \mu \mathrm{m}$-thick electrolyte in the capillary, respectively. By comparing the intensity profiles of a flat-view image and an electrode image, the electrode absorbs approximately $1.2 \%$ of the photon intensity illuminated on it.

\section{Results and discussion}

To specify radiation damage under an in situ environment, the $\mathrm{Ge}_{0.9} \mathrm{Se}_{0.1}$ electrode was exposed to X-rays with a photon energy of $11.2 \mathrm{keV}$ during $\sim 12 \mathrm{~min}$ of TXM tomography measurement. Seven hundred and twenty-one TXM images were captured for $1 \mathrm{~s}$ of X-ray exposure at $0.25^{\circ}$ rotation increments over $180^{\circ}$. Fig. 1 shows TXM images at various rotation angles. The TXM images depict the $\mathrm{Ge}_{0.9} \mathrm{Se}_{0.1}$ particle cluster of the electrode in bright color with a pixel size of $37.7 \mathrm{~nm}$ (imaging resolution). Reconstruction of raw tomography data requires retaining the structure of the sample during $180^{\circ}$ rotation. Thus, the initial $\left(0^{\circ}\right)$ and final $\left(180^{\circ}\right)$ TXM images should be symmetric to reconstruct the electrode microstructure. However, the final TXM image shows large changes of $\mathrm{Ge}_{0.9} \mathrm{Se}_{0.1}$ particle cluster after $\sim 12$ min of X-ray exposure. Video S1 (see supporting information) clearly shows the following morphology changes of the electrode: (1) growing distances between particles, and (2) sudden particle dislocations (e.g. $\Delta x$ and $\Delta y$ in Fig. 1). The morphology changes are considered to be as a result of the radiation damage on the carbon-binder matrix of the irradiated electrode.



Figure 1

TXM images of a $\mathrm{Ge}_{0.9} \mathrm{Se}_{0.1}$ electrode in in situ experimental environment at different angles during tomography under $11.2 \mathrm{keV}$ photon energy. The blue dashed lines at $0^{\circ}$ and $180^{\circ}$ show particle cluster changes after $12 \mathrm{~min}$ of irradiation. The $180^{\circ}$ image was horizontally flipped to directly compare with the $0^{\circ}$ image. $\Delta x$ and $\Delta y$ show sudden particle dislocation due to the change of the carbon-binder matrix.

To investigate the effect of radiation dose on the carbonbinder matrix, a $\mathrm{Ge}_{0.9} \mathrm{Se}_{0.1}$ electrode in electrolyte environment was exposed to $11.2 \mathrm{keV} \mathrm{X}$-rays and monitored by continuously capturing TXM images with $1 \mathrm{~s}$ exposure for $30 \mathrm{~min}$ without sample rotation. Fig. 2(a) shows $1 \mathrm{~s}$ and $30 \mathrm{~min}$ irradiated $\mathrm{Ge}_{0.9} \mathrm{Se}_{0.1}$ clusters and Video S2 shows the dynamic change. The TXM images clearly show the change of particle clusters during $30 \mathrm{~min}$ of irradiation. To quantify the radiation damage, two of the particle distances [marked as $L_{1}$ and $L_{2}$ in Fig. 2(a)] were tracked by the irradiation time. In Fig. 2(b), the onset of distance increment $\left(\Delta L_{1}\right)$ appeared at 6 min and $\Delta L_{1}$ reached $5.4 \%$ of the initial distance after $30 \mathrm{~min}$ of irradiation. The distance increment $\left(\Delta L_{2}\right)$ shows a similar pattern to $\Delta L_{1}$, but its onset appeared at $4 \mathrm{~min}$ and is $9.4 \%$ of the initial distance after $30 \mathrm{~min}$ of irradiation. The faster and larger change of $L_{2}$ is due to the higher X-ray dose in the middle of the illumination field. To test whether the damage on the carbon-binder matrix is primarily from free radicals released from the absorption of photons by $\mathrm{Ge}$, the same experiment was conducted at $11.2 \mathrm{keV}$ (after Ge $K$-edge) and $11 \mathrm{keV}$ (before Ge $K$-edge). As the $\mathrm{Ge}_{0.9} \mathrm{Se}_{0.1}$ particle size is about $2 \mu \mathrm{m}$, the X-ray absorption of $\mathrm{Ge}_{0.9} \mathrm{Se}_{0.1}$ particles at $11.2 \mathrm{keV}$ is about six times the absorption at $11 \mathrm{keV}$. In order to only compare the impact of the energy level, two regions on one wire $\mathrm{Ge}_{0.9} \mathrm{Se}_{0.1}$ electrode with similar dimensions were selected. Fig. S1 and Videos S3 and S4 show that the expansion of the $\mathrm{Ge}_{0.9} \mathrm{Se}_{0.1}$ electrode at two energy levels is similar, which demonstrates that the damage is primarily from the absorption of photons by the carbon-binder matrix.

We believe that synchrotron $\mathrm{X}$-ray radiation can have two effects on the polymer binder: scission and crosslinking (Coffey et al., 2002). Scission of a long-chain polymer generates short-chain polymers, which could be dissolved by the electrolyte. Therefore, some $\mathrm{Ge}_{0.9} \mathrm{Se}_{0.1}$ particles could be suddenly dislocated from the particle cluster. Crosslinking of 

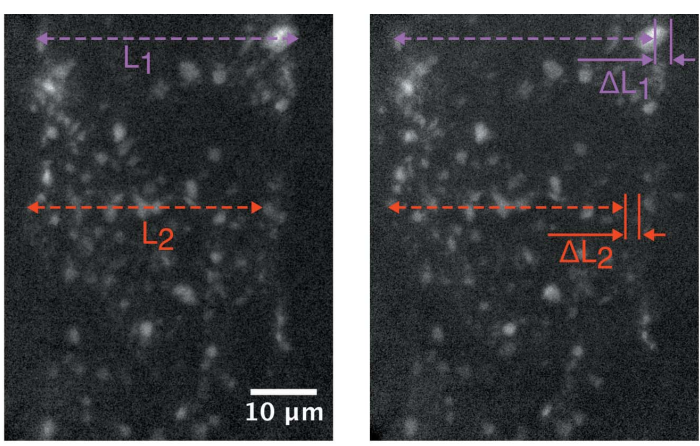

(a)

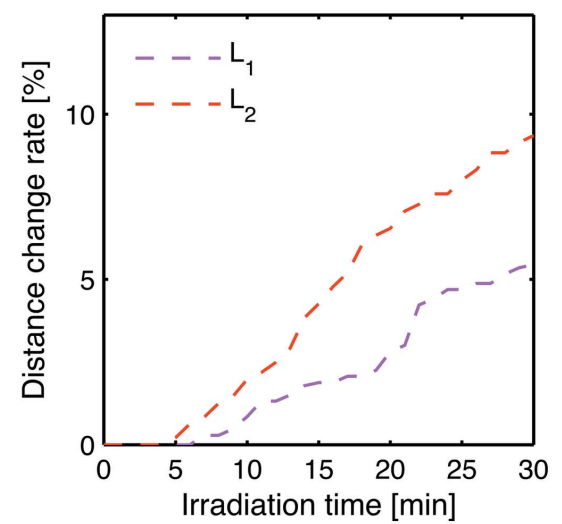

(b)

Figure 2

A series of TXM images with $1 \mathrm{~s}$ exposure are captured to monitor radiation damage on a $\mathrm{Ge}_{0.9} \mathrm{Se}_{0.1}$ electrode in electrolyte environment under $11.2 \mathrm{keV}$ photon energy. The TXM images show (a) $1 \mathrm{~s} \mathrm{(left)} \mathrm{and}$ $30 \mathrm{~min}$ (right) irradiated $\mathrm{Ge}_{0.9} \mathrm{Se}_{0.1}$ particle cluster with $37.7 \mathrm{~nm}$ pixel resolution. $(b)$ The increment of particle distances is tracked by the accumulated X-ray dose.

the polymer could cause swelling of the polymer in electrolyte, which is the reason for the expansion of the carbon-binder matrix. The distance tracking results indicate that the carbonbinder matrix maintains the $\mathrm{Ge}_{0.9} \mathrm{Se}_{0.1}$ particle cluster under the $11.2 \mathrm{keV} \mathrm{X}$-rays for $4 \mathrm{~min}$. Thus, the irradiation dose can be reduced to sustain the structure during TXM tomography. However, reducing the total dose means decreasing the number of images or the exposure time. Both will reduce the quality of tomography. Typically, tomography collects a series of TXM images under continuous X-ray exposure. For instance, the $\mathrm{Ge}_{0.9} \mathrm{Se}_{0.1}$ electrode has about $6 \mathrm{~min}$ of total $\mathrm{X}$-ray exposure if 361 images with $1 \mathrm{~s}$ exposure and $180^{\circ}$ rotation are used. The $6 \mathrm{~min}$ of continuous X-ray illumination can cause a change to the structure of the electrode by the damaged carbon-binder matrix. Vaselabadi et al. (2016) showed that radiation damage on polymers is likely to have an onset dose. If intermittent exposure was implemented with each exposure dose smaller than the onset dose and a long enough interval time between exposures, it is hypothesized that the radiation damage can be significantly reduced or avoided.

To test this hypothesis, X-rays were exposed for $1 \mathrm{~s}$ to capture an image and blocked by a shutter for 3-8 s before capturing the next image during in situ TXM tomography.

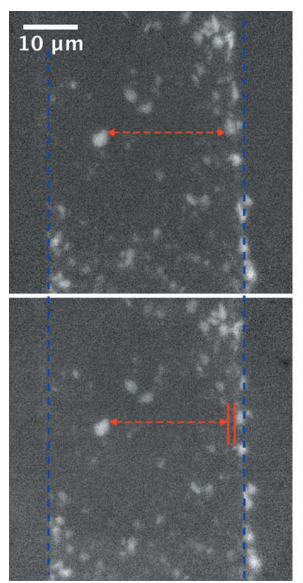

(a)

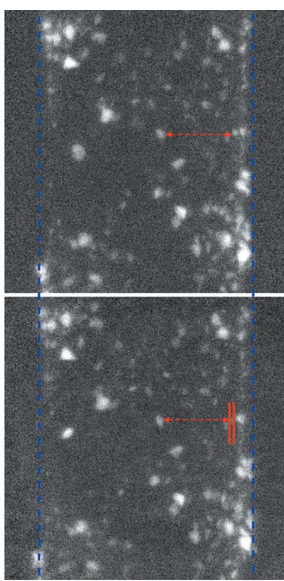

(b)

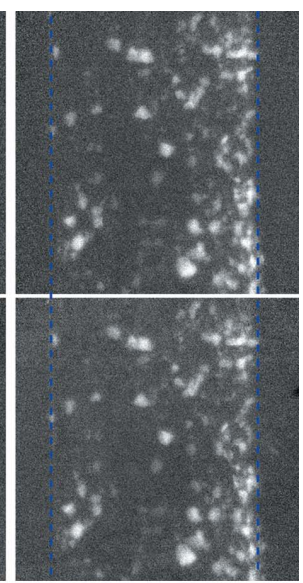

(c)
Figure 3

In situ TXM tomography of a $\mathrm{Ge}_{0.9} \mathrm{Se}_{0.1}$ electrode under intermittent $11.2 \mathrm{keV}$ photon energy. TXM images show the $\mathrm{Ge}_{0.9} \mathrm{Se}_{0.1}$ particle cluster with a $37.7 \mathrm{~nm}$-pixel resolution at the initial position $\left(0^{\circ}\right.$, top) and the horizontally flipped image at the last position $\left(180^{\circ}\right.$, bottom). The series of TXM images with $1 \mathrm{~s}$ exposure are obtained with $(a) 3 \mathrm{~s},(b) 5 \mathrm{~s}$ and (c) $8 \mathrm{~s}$ blocking time. The red marks indicate particle distance increments (a) $5.3 \%$ and (b) $2.6 \%$ after the TXM tomography.

Fig. 3 shows the initial and horizontally flipped last images of the TXM tomography with three different X-ray blocking times $(3,5$ and $8 \mathrm{~s})$. The dashed lines were drawn based on the initial TXM images to show the particle cluster changes after the tomography. The biggest increment of particle distance is around $5.3 \%$ with the $3 \mathrm{~s}$ blocking time shown in Fig. 3(a) and it is around $2.6 \%$ with the $5 \mathrm{~s}$ blocking time shown in Fig. 3(b). As shown in Fig. 3(c), the $8 \mathrm{~s}$ blocking time allows the particle cluster to be maintained during the in situ TXM tomography. The series of TXM images demonstrate the stable electrode structure during the tomography with $8 \mathrm{~s}$ blocking time in Video S5. The result suggests that radiation-induced morphology changes in in situ TXM tomography could be avoided by minimizing the accumulated $\mathrm{X}$-ray dose and choosing intermittent $\mathrm{X}$-ray exposure. It should be noted that radiation damage on the carbon-binder matrix could already start before the morphology change is detected at $4 \mathrm{~min}$ of continuous exposure. The undetected changes can de-activate some $\mathrm{Ge}_{0.9} \mathrm{Se}_{0.1}$ particles by breaking electronic connection. Radiation damage on the carbon-binder matrix depends on many factors, such as polymer materials, electrolyte, photon energy, photon rate, etc. The dose plan should be set based on the in situ experimental conditions. Another option to avoid the impact of radiation damage on the carbon-binder matrix is to use other binding techniques instead of a polymer binder. For instance, Wang et al. (2014) directly deposited Sn particles on a carbon substrate and conducted in situ TXM tomography successfully.

\section{Conclusions}

Radiation-induced damage of LIB electrodes was investigated in electrolyte environment for in situ TXM tomography. 
Particle cluster changes of the irradiated electrode revealed radiation damage on the carbon-binder matrix. Irradiation of the carbon-binder matrix induces scission and crosslinking of the polymer, which causes the dislocation of active material particles and an expansion of the carbon-binder matrix. The radiation damage on the carbon-binder matrix during TXM tomography can be avoided by minimizing the total irradiation dose and choosing intermittent X-ray exposure.

\section{Acknowledgements}

This work used resources of the Advanced Photon Source, a US Department of Energy (DOE) Office of Science User Facility operated for the DOE Office of Science by Argonne National Laboratory under contract No. DE-AC02$06 \mathrm{CH} 11357$.
Funding information

Funding for this research was provided by: US National Science Foundation (award No. 1335850).

\section{References}

Coffey, T., Urquhart, S. \& Ade, H. (2002). J. Electron Spectrosc. Relat. Phenom. 122, 65-78.

Klavetter, K. C., Pedro de Souza, J., Heller, A. \& Mullins, C. B. (2015). J. Mater. Chem. A, 3, 5829-5834.

Lim, C., Yan, B., Kang, H., Song, Z., Lee, W. C., De Andrade, V., De Carlo, F., Yin, L., Kim, Y. \& Zhu, L. (2016). J. Power Sources, 328, 46-55.

Nelson, J., Yang, Y., Misra, S., Andrews, J. C., Cui, Y. \& Toney, M. F. (2013). Proc. SPIE, 8851, 88510B.

Vaselabadi, S. A., Shakarisaz, D., Ruchhoeft, P., Strzalka, J. \& Stein, G. E. (2016). J. Polym. Sci. B, 54, 1074-1086.

Wang, J., Chen-Wiegart, Y. K. \& Wang, J. (2014). Angew. Chem. 126, 4549-4553.

Weker, J. N., Liu, N., Misra, S., Andrews, J., Cui, Y. \& Toney, M. (2014). Energy Environ. Sci. 7, 2771-2777. 